\title{
Distúrbios do metabolismo glicídico na síndrome da apneia obstrutiva do sono
}

\author{
Felipe de O. Figueiredo, ${ }^{1 *}$ Maria Lucia E. Pires, ${ }^{2}$ Lucas N. A. Lemes ${ }^{3}$
}

\begin{abstract}
Resumo
A síndrome da apneia obstrutiva do sono (SAOS), condição mais comum dentre os distúrbios relacionados ao sono, ocorre devido à obstrução repetida, intermitente, das vias aéreas superiores durante o sono com completa (apneia) ou parcial (hipopneia) interrupção do fluxo aéreo por 10 segundos ou mais. Estes episódios causam hipoxemia intermitente crônica (HIC) e fragmentação do sono que podem levar a distúrbios no metabolismo da glicose por vários mecanismos, incluindo a ativação do sistema nervoso simpático e do eixo hipotálamo-hipófise-adrenal; ativação do estresse oxidativo e de fatores pró-inflamatórios, como IL-6e TNF-alfa; diminuição dos níveis de adiponectina (e, postula-se, aumento dos níveis de leptina), assim como diminuição da secreção de insulina pelas células $\beta$ pancreáticas. Dessa forma, a SAOS pode ser considerada um fator de risco para desenvolvimento de resistência insulínica, intolerância à glicose e diabetes mellitus tipo 2. Este artigo se propõe a discutir as evidências relatadas na literatura quanto da existência de relação causal independente da apneia do sono com alterações do metabolismo glicídico.
\end{abstract}

Descritores: Sono; Apneia do sono tipo obstrutiva; Transtornos do metabolismo da glucose, fisiopatologia; Diabetes mellitus tipo 2 .

\section{Abstract \\ Glucose metabolism disorders in obstructive sleep apnea syndrome}

The obstructive sleep apnea syndrome (OSAS) is the most common condition among sleep-related disorders and occurs due to repeated, intermittent obstruction of the upper airway during sleep, with complete (apnea) or partial (hypopnea) airflow interruption. These episodes cause hypoxia and are associated with glycolipidics changes, formation of free radicals and oxidative stress, being a risk factor for development of insulin resistance, glucose intolerance, type 2 diabetes mellitus and metabolic syndrome. This article aims to discuss if there is relation between apnea and abnormal glucose metabolism.

Keywords: Sleep; Obstructive sleep apnea; Glucose Metabolism Disorders, physiopathology; Diabetes mellitus type 2.
1. Programa de Pós-graduação em Neurologia. Universidade Federal do Estado do Rio de Janeiro. Rio de Janeiro, RJ, Brasil.

2. Escola de Medicina e Cirurgia. Universidade Federal do Estado do Rio de Janeiro. Rio de Janeiro, RJ, Brasil.

3. Serviço de Otorrinolaringologia. Departamento de Especialidades Cirúrgicas. Faculdade de Ciências Médicas. Universidade do Estado do Rio de Janeiro. Rio de Janeiro, RJ, Brasil.

*Endereço para correspondência:

PPGNEURO, UNIRIO

Rua Mariz e Barros, 775

Rio de Janeiro, RJ, Brasil. CEP: 20270-004

E-mail: mestneuro@gmail.com

Revista HUPE, Rio de Janeiro, 2016;15(1):61-67

doi: 10.12957/rhupe.2016.22376

Recebido em 06/01/2015. Aprovado em 21/03/2016.

\section{Resumen}

Trastornos del metabolismo de la glucosa en el síndrome de apnea obstructiva del sueño

El síndrome de apnea obstructiva del sueño(SAOS), la condición más común entre los trastornos relacionados con el sueño, se produce debido a la obstrucción repetida, intermitente de la vía aérea superior durante el sueño con completa (apnea) o (hipopnea) interrupción parcial el flujo de aire durante 10 segundos o más. Estos episodios causan hipoxemia intermitente y sueño fragmentado puede conducir a perturbaciones en el metabolismo de la glucosa por diversos mecanismos, incluyendo la activación del sistema nervioso simpático y el eje hipotálamo-pituitaria-adrenal; la activación de factores de estrés oxidativo y proinflamatorias tales como IL-6 y TNF-alfa; disminución de los niveles de adiponectina (y se postula, el aumento de los niveles de leptina), y la disminución de la secreción de insulina de las células beta pancreáticas. Por lo tanto, la OSA puede ser considerado un factor de riesgo para el desarrollo de resistencia a la insulina, intolerancia a la glucosa y diabetes mellitus tipo 2 (DM2). Este artículo tiene como objetivo discutir la evidencia reportada en la literatura sobre la existencia de una relación causal independiente de la apnea del sueño con el metabolismo anormal de la glucosa.

Palabras clave: Sueño; Apnea del sueño obstructiva; Glucose metabolism disorders, fisiopatología; Diabetes mellitus tipo 2 . 


\section{Introdução}

A síndrome da apneia obstrutiva do sono (SAOS) manifesta-se como uma redução (hipopneia) ou cessação (apneia) do fluxo aéreo, apesar do esforço inspiratório, com duração de, pelo menos, 10 segundos. ${ }^{1}$ Episódios recorrentes de obstrução das vias aéreas superiores durante o sono provocam hipóxia intermitente crônica (HIC) e fragmentação do sono, em um processo de breves despertares e ciclos de hipoxemia/ reoxigenação tecidual. Este quadro ativa o sistema nervoso simpático e o eixo hipotálamo-hipófise-adrenal no período de sono, levando a um estresse oxidativo e inflamação sistêmica que tendem a gerar alterações metabólicas e resultam no aparecimento ou exacerbação de anormalidades pré-existentes, como resistência à insulina, diabetes mellitus (DM) tipo 2, hipertensão arterial sistêmica (HAS) e um dano endotelial vascular, tudo isso favorecendo o desenvolvimento de doenças cardiovasculares (DCV).,3

A obesidade com deposição de gordura visceral é um elemento comum tanto na SAOS quanto da Síndrome Metabólica. Sabe-se que a obesidade central está associada a um fator de risco independente para intolerância à glicose e resistência insulínica. Embora esta obesidade seja um fator de risco para ambas as condições, há evidências na literatura de que a apneia do sono seja um fator de risco independente para a intolerância à glicose e a resistência à insulina, mas são necessários mais estudos bem delineados que demonstrem se as alterações metabólicas encontradas nos pacientes com apneia do sono independem da quantidade de gordura corporal. ${ }^{4}$

ASAOS é uma condição comum e frequentemente subestimada. ${ }^{5}$ Há uma disparidade entre a presença oculta da SAOS e a baixa prevalência de casos confirmados na população, sendo referido que a síndrome clinicamente grave não está diagnosticada em $82 \%$ dos homens e $92 \%$ das mulheres. ${ }^{6}$ Há grande variabilidade na prevalência da SAOS em estudos com adultos, em grande parte devido às diferenças conceituais da doença e dos parâmetros de mensuração. Estudos iniciais observaram a presença de DRS clinicamente importante ocorrendo em $2 \%$ das mulheres e $4 \%$ dos homens entre 30 e 60 anos de idade. ${ }^{7}$ No entanto, quando o diagnóstico da SAOS se baseia na polissonografia (PSG) noturna, tem sido observada prevalência de até $9 \%$ em mulheres e $24 \%$ em homens. ${ }^{8}$ Um estudo epidemiológico recente demonstrou que $20 \%$ dos adultos de meia-idade têm, pelo menos, SAOS leve. ${ }^{5}$ Outro estudo realizado na cidade de São Paulo, utilizando para diagnóstico a PSG, observou alta prevalência da SAOS $(32,8 \%)$ na população adulta dessa cidade.,10

Indivíduos com SAOS têm uma alta prevalência de DM tipo 2 e desordens metabólicas relacionadas. Há uma proporção substancial de indivíduos com DM tipo 2 que sofre deSAOS não reconhecida, mas naqueles com SAOS conhecida estima-se que a prevalência de DM2 varia de 29\% a 63\%, dependendo dos critérios adotados. ${ }^{11,12}$ A SAOS é ainda mais elevada (>86\%) nos indivíduos obesos com diabetes. ${ }^{13}$ A principal hipótese de esta interrelação favorecer o DM tipo 2 se associa a um aumento da resistência insulínica, sendo demonstrado que a duração e a qualidade do sono são indicativas do controle glicêmico nos diabéticos. ${ }^{14}$ Entretanto, apesar destas evidências epidemiológicas, os resultados dos estudos são controversos, sendo que alguns postulam que a SAOS predispõe à resistência insulínica independentemente da obesidade, enquanto outros sugerem a influência da obesidade no surgimento desta resistência à insulina. Assim sendo, o papel da SAOS no manejo do DM tipo 2 necessita ser melhor compreendido. ${ }^{15}$

\section{SAOS, resistência insulínica e diabetes mellitus tipo 2}

Estudos inicialmente realizados sobre a relação de SAOS e RI em pacientes com SAOS indicaram que os níveis aumentados de insulina ou a resistência aos seus efeitos exprimiam os efeitos da obesidade concomitante. ${ }^{16,17}$ No entanto, estudos posteriores mostraram que pacientes com SAOS apresentam níveis mais elevados de glicemia de jejum, insulina e hemoglobina A1C mesmo quando comparados com indivíduos do mesmo peso e sem SAOS. ${ }^{18}$ Estas associações foram verificadas em estudos em indivíduos com peso normal, independentemente da idade ou do índice de massa corpórea. ${ }^{19,20} \mathrm{Em}$ outros estudos, o grau de resistência à insulina correlacionou-se diretamente tanto com a severidade da SAOS pelo índice de apneia e hipopneia (IAH) como com períodos prolongados de hipóxia noturna, mesmo em não diabéticos. ${ }^{1,21}$ Um recente estudo prospectivo verificou que elevados níveis de insulinemia precediam a SAOS em indivíduos cujos episódios de apneia já haviam sido presenciados por terceiros. ${ }^{15}$

A SAOS tem sido assim apontada como um fator de risco para o aparecimento de DM tipo $2 .{ }^{21} \mathrm{Um}$ estudo mostrou que indivíduos com SAOS, com IAH superior a 20, apresentavam um risco de diabetes mellitus cinco vezes superior ao de indivíduos sem SAOS, de forma independente da presença de obesidade central. ${ }^{18}$ Há 


\section{Artigo de revisão}

cada vez mais evidências que suportam uma associação independente entre SAOS e alterações do metabolismo glicídico. 8,22,23 Em humanos saudáveis, a hipóxia induziu intolerância à glicose e aumento da concentração plasmática de adrenalina. ${ }^{24}$ Estudos em modelos animais identificaram que a hipóxia intermitente crônica da apneia do sono resulta em aumento da resistência insulínica tanto em ratos geneticamente obesos quanto nos magros. ${ }^{25,26}$

Dois estudos clássicos mostraram a relação entre SAOS, resistência insulínica e DM tipo 2 independente da obesidade. O Sleep Heart Health Study ${ }^{27}$ mostrou uma significante associação entre dessaturação do oxigênio durante o sono e elevada concentração de glicose plasmática de jejum de $2 \mathrm{~h}$ durante um teste oral de tolerância à glicose. A severidade da SAOS foi também associada ao grau de resistência insulínica após ajuste para obesidade. O Wisconsin Sleep Study, ${ }^{28} \mathrm{com}$ 1.387 participantes, estudou a associação entre apneia do sono e DM tipo 2. Foi um estudo seccional e longitudinal que estimou a prevalência e a incidência de DM tipo 2 em indivíduos com SAOS. A associação foi preservada naqueles com SAOS moderada a severa após ajuste para obesidade (OR $=2,3)$, entretanto, os dados longitudinais do mesmo estudo não mostraram ser a SAOS um significante preditor de desenvolvimento de DM tipo 2 no período de quatro anos. Os pesquisadores concluíram que diabetes é mais prevalente na SAOS e sua relação com DM tipo 2 é independente de outros fatores de risco. Entretanto, não está claro se SAOS é causal no desenvolvimento de diabetes.

O tratamento com pressão continua e positiva das vias aéreas (CPAP) em relação à resistência insulínica mostra resultados controversos. Alguns estudos relatam que CPAP não reverte resistência insulínica de forma aguda, enquanto outros observaram essa reversão imediata. ${ }^{18,21}$ Os efeitos no longo prazo verificaram melhorias no metabolismo da glicose e na resistência à insulina ao fim de 4 meses e de 7,5 meses de tratamento, apesar de o peso corporal ter se mantido estável. Assim, compreende-se que a SAOS por si só seja um fator de risco independente para a resistência à insulina.

\section{Mecanismos implicados na patogênese da SAOS}

Um crescente número de estudos sugere que a SAOS está envolvida na patogênese de distúrbios do metabolismo glicídico. ${ }^{29,30}$ Existem evidências de que o estresse fisiológico imposto pela HIC (hipóxia do tecido/reoxigenação), ${ }^{8,21,31}$ privação do sono ou frag- mentação por microdespertares frequentes típica da SAOS $^{32}$ e a curta duração do sono, ${ }^{21}$ no longo prazo, podem estar envolvidos na patogênese dos distúrbios no metabolismo da glicose por várias vias, incluindo a ativação do sistema nervoso simpático e do eixo hipotálamo-hipófise-adrenal, ativação do estresse oxidativo e de fatores pró-inflamatórios, como IL-6 e TNF-alfa. ${ }^{2}$ Outros mecanismos incluem hipóxia pancreática e consequente diminuição da secreção de insulina pancreática, possíveis alterações centrais hipotalâmicas que participem do controle glicêmico e, talvez, aumento dos níveis de leptina (resistência à leptina)., ${ }^{4,14,18,33,34}$ Os potenciais mecanismos são descritos abaixo e esquematizados na figura 1 .

\section{Ativação do sistema nervoso simpático (SNS)}

O SNS desempenha um papel central na regulação do metabolismo da glicose e dos lipídios e contribui diretamente para a resistência insulínica. ${ }^{14}$ A SAOS tem mostrado aumentar a atividade simpática não somente durante o sono, mas também acordado. A ativação simpática parece ser predominantemente um resultado da hipóxia noturna, entretanto, o repetido despertar do sono que segue cada evento respiratório obstrutivo parece também exacerbar esse efeito. ${ }^{35}$ Esses mecanismos ativam o SNS, que é um potente estimulador da lipólise e lipotoxicidade mediadas pelas catecolaminas sistêmicas liberadas. Os ácidos graxos livres resultantes da lipólise reduzem a captação de glicose mediada pela insulina, devido à interrupção da sinalização insulínica no músculo esquelético e causam disfunção da célula beta pela lipotoxicidade. Além disso, as catecolaminas estimulam diretamente a mobilização do glicogênio e inibem a captação de glicose pelo músculo, estimulam a secreção de glucagon, inibem a secreção de insulina e aumentam a gliconeogênese e, principalmente, a glicogenólise no fígado. 8,21,33,36

\section{Disfunção do eixo hipotálamo- hipofisário-adrenal (HHA)}

O cortisol inibe a secreção insulínica e a HIC com fragmentação do sono ativam o eixo HHA resultando na elevação dos níveis de glicocorticoides que têm efeitos diretos na resistência insulínica e na secreção de insulina. ${ }^{8,14,21}$ Esses efeitos incluem aumento da lipólise, inibição da translocação do transportador de glicose tipo 4 (GLUT 4) dependente de insulina nas células musculares, supressão da síntese de glicogênio e aumento da gliconeogênese. 


\section{Inflamação sistêmica}

A HIC em pacientes com SAOS pode levar a insulinoresistência e intolerância à glicose por ativação da resposta inflamatória sistêmica, com consequente aumento da liberação de citocinas pró-inflamatórias e ativação de linfócitos e monócitos, com evidências de que essas alterações são independentes de adiposidade., ${ }^{2,14,21,27}$ A maior parte desses efeitos é devida à HIC, mas a ativação simpática provavelmente também desempenha um papel. ${ }^{8}$ Estes pacientes apresentam níveis elevados de proteína C-reativa independentemente da obesidade, sendo os níveis desta proteína proporcionais à gravidade da SAOS. ${ }^{18}$ Os níveis plasmáticos de IL-6 e de TNF-alfa são maiores em pacientes com SAOS em relação aos indivíduos do grupo controle, com incrementos dos valores de IL-6 intimamente correlacionados com aumento da resistência insulínica. ${ }^{37}$

Há evidências de que as citocinas inflamatórias TNF-alfa, IL-1 $1 \beta$ e IL-6 estão envolvidas na fisiologia da regulação do sono. A sua secreção ou administração exógena em humanos está associada à sonolência e fadiga. Os estudos realizados para a IL-6 e TNF-alfa indicam que estes têm um papel importante na mediação dos principais sintomas da SAOS. O tratamento com CPAP, durante um mês, reduz substancialmente os níveis de proteína C-reativa e IL-6. ${ }^{18}$

Consideradas em conjunto, as informações atualmente disponíveis indicam que a HIC pode levar à intolerância à glicose e à resistência insulínica, promovendo a liberação de citocinas pró-inflamatórias, tais como interleucina-6 (IL-6) e fator de necrose tumoral-alfa (TN$\mathrm{F}-$ alfa), ${ }_{,}^{27}$ com papel significativo no desenvolvimento de disfunção metabólica.

\section{Adipocinas}

HIC pode levar à resistência insulínica por alterar a produção de adipocinas, hormônios produzidos no tecido adiposo. Níveis de leptina têm se mostrado mais elevados e de adiponectina mais baixos nos pacientes com SAOS. Leptina é uma citocina produzida no tecido adiposo e está envolvida no controle do peso corporal e em uma variedade de funções biológicas. Níveis séricos de leptina são correlacionados com IMC e IAH, provavelmente devido à baixa tensão de oxigênio celular típica da SAOS, e aparentemente melhorando com CPAP.

Um maior IAH aumenta a expressão do gene da leptina e os níveis séricos dessa proteína. A leptina atua em nível central e periférico, inibindo a secreção insulínica. ${ }^{38} \mathrm{O}$ desenvolvimento de resistência insulínica durante HIC na presença de hiperleptinemia pode sugerir resistência leptínica. Finalmente, hipoxemia intermitente pode suprimir secreção de adiponectina, um hormônio que aumenta a sensibilidade à insulina. ${ }^{8,21}$

Um estudo em modelos animais utilizando a HIC no desenvolvimento de resistência insulínica observou aumento dos níveis de leptina em animais magros e aumento da secreção de insulina em animais com deficiência na produção de leptina, efeito este abolido pela infusão exógena de leptina. Estes autores concluem que a resistência insulínica da HIC se deve a uma alteração da ação fisiológica da leptina. ${ }^{26}$

\section{Estresse oxidativo}

A obstrução das vias aéreas superiores leva à diminuição da oxigenação do sangue e HIC, caracterizada por episódios repetidos de hipóxia e reoxigenação. A geração de radicais livres nas células durante a hipóxia foi relatada como semelhante ao processo de isquemia/ reperfusão de sangue, quando ocorre redução do potencial redox mitocondrial, causando uma produção de espécies reativas de oxigênio a partir da cadeia de transporte de elétrons, principalmente no nível $3 \mathrm{do} \mathrm{ci-}$ tocromo. O estresse oxidativo induzido pela HIC parece ser a primeira causa de dano no organismo na SAOS. A HIC induz a geração de espécies reativas de oxigênio, provavelmente devido à depleção repetida de oxigênio seguida de hiperoxigenação para restaurar a pressão de oxigênio $\left(\mathrm{PO}^{2}\right)$. Estudos recentes sugerem que a HICleva ao estresse oxidativo e ativação da resposta inflamatória sistêmica, com aumento da atividade antioxidante no sangue e na produção de citocinas inflamatórias. Em pacientes diabéticos, as espécies reativas de oxigênio prejudicam a captação de glicose no músculo e gordura e leva à diminuição da secreção de insulina pelas células beta pancreáticas. ${ }^{39}$

\section{Hipóxia intermitente crônica (HIC) e célula beta $(\beta)$ pancreática}

Diabetes é a manifestação final da resistência insulínica e insuficiente secreção da insulina compensatória da célula $\beta$ pancreática. Evidências experimentais sobre o efeito da HIC nas células $\beta$ pancreáticas são escassas. A imunohistoquímica das ilhotas pancreáticas mostrou que HIC, em curto prazo, aumenta apoptose e proliferação de células $\beta$ pancreáticas. A superexpressão da enzima antioxidante superóxido desmutase em ilhotas pancreáticas as protege contra a apoptose induzida por HIC, enquanto a proliferação das células C induzida por 
HIC não foi afetada. ${ }^{38}$ Ao contrário, dados em voluntários saudáveis sugerem que HIC pode danificar a função das células $\beta$ pancreáticas. Assim, HIC induz resistência insulínica e pode ter efeito deletério na função endócrina pancreática. ${ }^{21,39}$

\section{Arquitetura do sono}

Um estudo recente examinou a supressão seletiva da fase 3 do sono não REM, um estágio do sono considerado ser restaurador em adultos jovens saudáveis, sem afetar a duração do sono ou causar hipóxia. A intervenção reduziu acentuadamente a sensibilidade à insulina e conduziu a um dano da tolerância à glicose. ${ }^{8}$

Estudos atuais avaliaram a possível interação entre DM tipo 1 e o sono. Pesquisadores observaram que diabéticos tipo 1 que tinham curta duração do sono (média $\leq 6,5$ horas) apresentavam hemoglobina glicada (HbA1c) significativamente mais elevada em relação aos que tinham maior duração do sono (>6 horas), ressaltando a importância da duração do sono como um elemento fundamental para a manutenção de um controle glicêmico adequado e prevenção de complicações crônicas. ${ }^{40}$

Outro estudo recente analisou a relação do sono com o controle glicêmico em indivíduos com DM tipo 1. Seus resultados suportam a hipótese de que existe uma interação entre parâmetros do sono e o controle glicêmico, no qual este desempenha um importante papel. Sugere, ainda, que exista um círculo vicioso envolvendo o sono e o controle glicêmico em indivíduos com DM tipo 1, de modo que um pobre controle glicêmico afetaria parâmetros do sono, e estes, quando alterados, comprometeriam ainda mais o controle da glicemia. Os pesquisadores sugerem que uma melhor manutenção dos níveis glicêmicos poderia melhorar a qualidade do sono. ${ }^{41}$

\section{Considerações finais}

Evidências atuais apoiam fortemente uma associação independente entre SAOS, resistência insulínica e intolerância à glicose, especialmente nas formas moderada e grave da doença. A relação de causalidade entre a SAOS e o desenvolvimento do diabetes mellitus tipo 2 permanece por ser esclarecida. Contudo, estudos apontam para a influência da duração e da qualidade do sono no risco e severidade do diabetes mellitus, tanto tipo 1 quanto tipo 2, sugerindo, inclusive, que esses parâmetros do sono sejam considerados como parte do tratamento do diabetes mellitus.

Quanto aos mecanismos fisiopatológicos propostos para explicar a ligação entre SAOS e distúrbios glicídicos, mais estudos são necessários antes que se possa inferir achados da pesquisa básica à prática clínica. Da mesma forma,são imprescindíveis estudos clínicos controlados randomizados de tratamento com CPAP com avaliações validadas quanto à sensibilidade periférica à insulina e quanto à tolerância à glicose para melhor compreensão sobre se o tratamento com CPAP melhora o metabolismo da glicose.

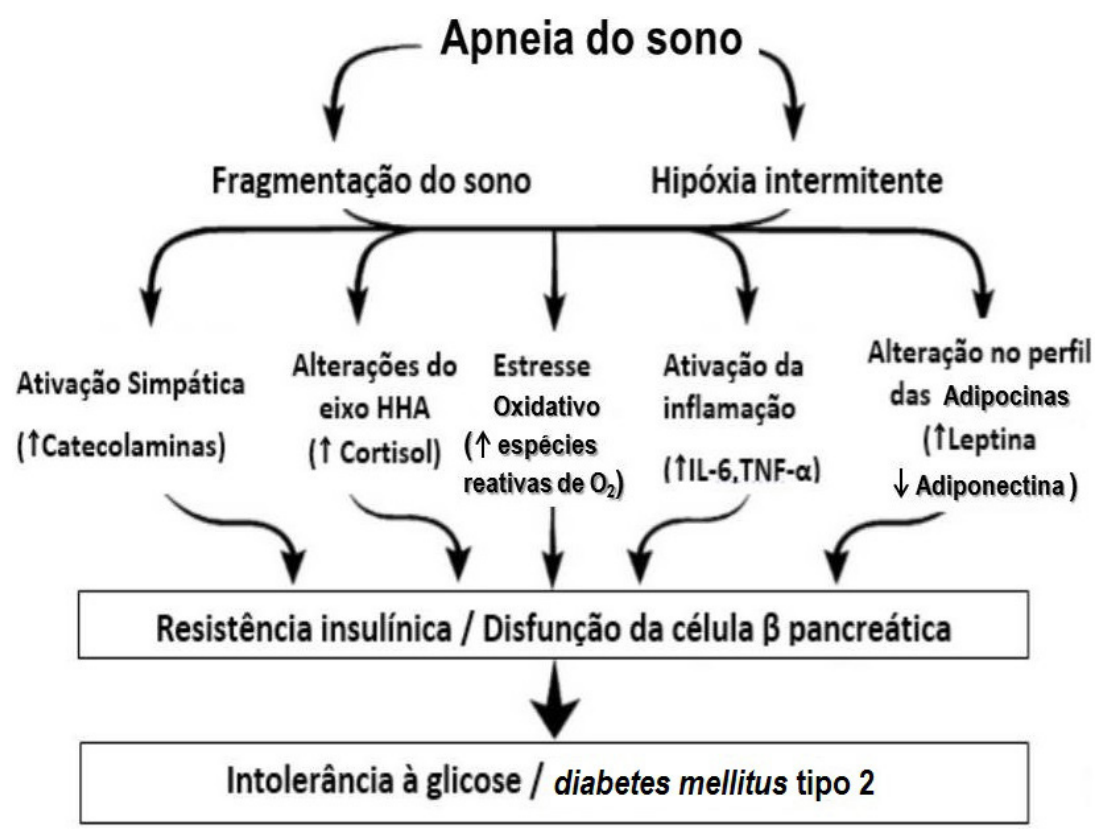

Figura 1. Mecanismos potenciais na relação SAOS e diabetes mellitus tipo 2. 
Pelo exposto, conclui-se pela necessidade de divulgação no meio científico e para os profissionais médicos, da associação de distúrbios respiratórios do sono e diabetes mellitus, especialmente o tipo 2, de forma a aumentar o diagnóstico, melhorar a prática clínica e coordenar os esforços de pesquisa para melhor entendimento da associação dos distúrbios respiratórios do sono com distúrbios do metabolismo glicídico, pois, enquanto a magnitude da epidemia de diabetes mellitus tipo 2 é bem reconhecida, distúrbios de respiração durante o sono não o são, apesar da significativa importância como comorbidade e do ônus financeiro imposto sobre os diversos países.

\section{Referências}

1. Ip MS, Lam B, Ng MM, et al. Obstructive Sleep Apnea is Independently Associated with Insulin Resistance. Am J Respir Crit Care Med. 2002;165:670-6. http://dx.doi.org/10.1164/ajrccm.165.5.2103001

2. Jun J, Polotsky VY. Metabolic consequences of sleep-disordered breathing. ILAR J. 2009;50:289-306.

3. Lemes L, Motta LCM, Lavorato FG, et al. Características clínicas dos distúrbios respiratórios do sono: intersecções epidemiológicas com a síndrome metabólica. Revista Hospital Universitário Pedro Ernesto. 2003;2(1):16-21.

4. Carneiro G, Fontes FH, Togueiro SMGP. Consequências metabólicas na SAOS não tratada. J Bras Pneumol. 2010;36(supl.2):43-6.

5. Gilat H, Vinker S, Buda I, et al. Obstructive Sleep Apnea and Cardiovascular Comorbidities A Large Epidemiologic Study. Medicine. 2014;93:9.

6. Chung F, Elsaid H. Screening for obstructive sleep apnea before surgery: why is it important? Curr Opin Anaesthesiol. 2009;22:405-11. http://dx.doi.org/10.1097/ACO.0b013e32832a96e2

7. Young T, Palta M, Dempsey J, et al. The Occurrence of Sleep-Disordered Breathing among Middle-Aged Adults. N Engl J Med. 1993;328:1230-5. http://dx.doi.org/10.1056/ NEJM199304293281704

8. Shaw JE, Punjabi NM, Wilding JP, et al. International Diabetes Federation Taskforce on Epidemiology and Prevention. Sleep-disordered breathing and type 2 diabetes: a report from the International Diabetes Federation Taskforce on Epidemiology and Prevention. Diabetes Res Clin Pract. 2008 Jul;81(1):212. http://dx.doi.org/10.1016/j.diabres.2008.04.025

9. Tufik S, Santos-Silva R, Taddei JA, et al. Obstructive Sleep Apnea Syndrome in the Sao Paulo Epidemiologic Sleep Study. Sleep Med. 2010;11:441-6. http://dx.doi.org/10.1016/j. sleep.2009.10.005

10. Zancanella E, Haddad F, Oliveira L, et al. Obstructive sleep apnea and primary snoring: diagnosis. Braz J Otorhinolaryngol. 2014;80(1 Suppl 1):1-16.

11. Einhorn D, Stewart DA, Erman MK, et al. Prevalence of sleep apnea in a population of adults with type 2 diabetes mellitus. Endocr Pract. 2007;13:355-62. http://dx.doi.org/10.4158/ EP.13.4.355

12. Laaban JP, Daenen S, Léger D, et al. Prevalence and predictive factors of sleep apnoea syndrome in type 2 diabetic patients.
Diabetes Metab. 2009;35:372-7. http://dx.doi.org/10.1016/j. diabet.2009.03.007

13. Foster GD, Sanders MH, Millman R, et al. Sleep AHEAD Research Group. Obstructive sleep apnea among obese patients with type 2 diabetes. Diabetes Care. 2009;32:1017-9.

14. Fusetti M, Fioretti AB, Valenti M, et al. Cardiovascular and metabolic comorbidities in patients with obstructive sleep apnea syndrome. Acta Otorhinolaryngologica Italica. 2012;32(5):320-5.

15. Balkau B, Vol S, Loko S, et al. Epidemiologic Study on the Insulin Resistance Syndrome Study Group. High baseline insulin levels associated with 6-year incident observed sleep apnea. Diabetes Care. 2010;33(5):1044-9. http://dx.doi.org/10.2337/ dc09-1901

16. Davies RJ, Turner R, Crosby J, et al. Plasma insulin and lipid levels in untreated obstructive sleep apnea and snoring; their comparison with matched controls and response to treatment. J Sleep Res.1994;3(3):180-5. http://dx.doi. org/10.1111/j.1365-2869.1994.tb00126.x

17. Stoohs RA, Facchini F, Guilleminault C. Insulin resistance and sleep-disordered breathing in healthy humans. Am J Respir Crit Care Med. 1996;154(1):170-4. http://dx.doi.org/10.1164/ajrccm.154.1.8680675

18. Fernandes MJA. Síndrome de apneia obstrutiva do sono e obesidade. Revista da SPCNA. 2006;12(2):41-57.

19. Punjabi NM, Sorkin JD, Katzel LI, et al. Sleep-disordered Breathing and Insulin Resistance in Middle-aged and Overweight Men. Am J Respir Crit Care Med. 2002;165:677-682. http://dx.doi.org/10.1164/ajrccm.165.5.2104087

20. Meslier N, Gagnadoux F, Giraud P, et al. Impaired glucose-insulin metabolism in males with obstructive sleep apnea syndrome. Eur Respir J. 2003;22(1):156-160. http://dx.doi.org/10.1 183/09031936.03.00089902

21. Drager LF, Jun JC, Polotsky VY. Metabolic consequences of intermittent hypoxia: relevance to obstructive sleep apnea. Best practice \& research Clinical endocrinology \& metabolism. 2010;24(5):843-51. http://dx.doi.org/10.1016/j. beem.2010.08.011

22. Papanas N, Steiropoulos P, Nena E, et al. HbA1c is associated with severity of obstructive sleep apnea hypopnea syndrome in nondiabetic men. Vasc Health Risk Manag. 2009;5:751-6. http://dx.doi.org/10.2147/VHRM.S7057

23. Pamidi S, Aronsohn RS, Tasali E. Obstructive sleep apnea: role in the risk and severity of diabetes. Best Pract Res Clin Endocrinol Metab. 2010;24:703-715. http://dx.doi.org/10.1016/j. beem.2010.08.009

24. Oltmanns KM, Gehring H, Rundolf S, et al. Hypoxia causes glucose intolerance in humans. Am J Respir Crit Care Med. 2004;169:1231-7. http://dx.doi.org/10.1164/rccm.200308-12000C

25. liyori N, Alonso LC, Li J, et al. Intermittent hypoxia causes insulin resistance in lean mice independent of autonomic activity. Am J Respir Crit Care Med. 2007;175:851-7. http://dx.doi. org/10.1164/rccm.200610-1527OC

26. Polotsky VY, Li J, Punjabi NM, et al. Intermittent hypoxia increases insulin resistance in genetically obese mice. $J$ Physiol. 2003;552:253-64. http://dx.doi.org/10.1113/jphysiol.2003.048173

27. Punjabi NM, Shahar E, Redline S, et al. Sleep Heart Health Study Investigators. Sleep-disordered breathing, glucose intolerance, and insulin resistance: the Sleep Heart Health Study. Am J Epidemiol. 2004:160(6):521-30. http://dx.doi.org/10.1093/ aje/kwh261 
Artigo de revisão

28. Reichmuth KJ, Austin D, Skatrud JB, et al. Association of sleep apnea and type II diabetes: a population-based study. Am J Respir Crit Care Med. 2005;172(12):1590-5. http://dx.doi. org/10.1164/rccm.200504-6370C

29. Botros N, Concato J, Mohsenin V, et al. Obstructive Sleep Apnea as a risk factor for type 2 diabetes. Am J Med. 2009 Dec;122(12):1122-7. http://dx.doi.org/10.1016/j.amjmed.2009.04.026

30. Fendri S, Rose D, Myambu S, et al. Nocturnal hyperglycaemia in type 2 diabetes with sleep apnea syndrome. Diabetes Res Clin Pract. 2011;91(1):e21-3. http://dx.doi.org/10.1016/j. diabres.2010.09.029

31. Keckeis M, Lattova Z, Maurovich-Horvat E, et al. Impaired Glucose Tolerance in Sleep Disorders. PLoS ONE. 2010;5(3):e9444. http://dx.doi.org/10.1371/journal. pone.0009444

32. Lemes L. Efeitos antropométricos, clínicos, laboratoriais e polissonográficos do CPAP nasal por 12 semanas em pacientes com síndrome da apneia obstrutiva do sono de moderada a grave intensidade: associações com o índice de massa corporal, com a faixa etária e com a morfologia do pescoço. 2007;1:169p. Doutorado. Universidade do Estado do Rio De Janeiro - Biologia (Biociências Nucleares).

33. Punjabi NM, Polotsky VY. Disorders of Glucose Metabolism in Sleep Apnea. J Appl Phisiol. 2005;99:2008-19. http://dx.doi. org/10.1152/japplphysiol.00695.2005

34. Pamidi S, Tasali E. Obstructive sleep apnea and type 2 dia- betes: is there a link? Front Neurol. 2012;3:126. http://dx.doi. org/10.3389/fneur.2012.00126

35. Polotsky VY, Rubin AE, Balbir A, et al. Intermittent hypoxia causes REM sleep deficits and decreases EEG delta power in NREM sleep in the C57BL/6J mouse. Sleep Med. 2006 Jan;7(1):7-16. http://dx.doi.org/10.1016/j.sleep.2005.06.006

36. Tkacova R, Dorkova Z, Molcanyiova A, et al. Cardiovascular Risk and Insulin Resistance in Patients with Obstrutive Sleep Apnea. Med Sci Monit. 2008;14(9):438-44.

37. Vgontzas AN, Papanicolaou DA, Bixler EO, et al. Sleep apnea and daytime sleepiness and fatigue: relation to visceral obesity, insulin resistance, and hypercytokinemia. J Clin Endocrinol Metab. 2000 Mar;85(3):1151-8. http://dx.doi.org/10.1210/ jcem.85.3.6484

38. Xu J, Long YS, Gozal D, et al. Beta-cell death and proliferation after intermittent hypoxia: role of oxidative stress. Free Radic Biol Med. 2009;46(6):783-90.

39. Rosa DP. Hepatoproteção dos antioxidantes melatonina e n-acetilcisteína na hipóxia intermitente. 2013;1:140p. Doutorado. Universidade Federal do Rio Grande do Sul.

40. Borel AL, Pépin JL, Nasse L, et al. Short Sleep Duration Measured by Wrist Actimetry Is Associated With Deteriorated Glycemic Control in Type 1 Diabetes. Diabetes Care. 2013;36(10):2902-8. http://dx.doi.org/10.2337/dc12-2038

41. Barone MKU, Wey D, Schorr F, et al. Sleep and glycemic control in type 1 diabetes. Arch Endocrinol Metab. 2015 Fev;59(1):71-8. http://dx.doi.org/10.1590/2359-3997000000013 